\title{
Video See Through AR Head-Mounted Display for Medical Procedures
}

\author{
Fabrizio Cutolo*, Paolo Domenico Parchi, Vincenzo Ferrari \\ EndoCAS Center, Department of Translational Research and New Technologies in Medicine and Surgery University of Pisa, 56124 Pisa, Italy
}

\begin{abstract}
In the context of image-guided surgery (IGS), AR technology appears as a significant development in the field since it complements and integrates the concepts of surgical navigation based on virtual reality.

The aim of the project is to optimize and validate an ergonomic, accurate and cheap video see-through AR system as an aid in various typologies of surgical procedures.

The system will ideally have to be inexpensive and user-friendly to be successfully introduced in the clinical practice.

Keywords: Mixed / augmented reality, Image-guided surgery, Medical device validation, Interest point and salient region detections, Object detection, Tracking.

Index Terms: [Computing methodologies]: Computer graphics - Graphics systems and interfaces; [Applied computing]: Life and medical sciences - Imaging; [Computing methodologies]: Artificial intelligence - Computer vision - Image and video acquisition / Computer vision problems.
\end{abstract}

\section{Research Context and Motivation}

Augmented reality (AR) represents a cutting edge technology in the field of machine vision and computer graphics. It allows merger of data from the real environment with virtual information. The enrichment of the real scene may be simply informative (i.e. textual or numerical values relevant to what is under observation) or may consist of three-dimensional virtual objects inserted within the real environment in spatially defined positions [1]. In the context of image-guided surgery (IGS), AR technology appears as a significant development in the field, because it complements and integrates the concepts of surgical navigation based on virtual reality [2]. AR can provide the surgeon with an inner perception of where the virtual content, generally obtained by radiological images, is located within an actual scene [3].

$\mathrm{PhD}$ project is aimed at developing and clinically validating augmented reality (AR) applications as an aid in various typologies of surgical procedures.

Most of the work will be carried out at EndoCAS, center for computer assisted surgery affiliated to the Faculty of Medicine of the University of Pisa. PhD started in November 2012.

\section{BACKGROUND AND GOALS}

In recent years, tools (or defined applications) employing AR have been designed and tested in the context of several surgical and medical disciplines, including maxillofacial surgery [4],[5],[6]; head and neck surgery [7]; neurosurgery [8],[9]; orthopedic surgery $[10],[11],[12]$ and general surgery [13],[14],[15],[16].

Nevertheless, as is true of many emerging technologies, no standard method by which AR technology could/should be transferred to clinical practice has yet been developed [17].

$\mathrm{PhD}$ work will be oriented to obtaining a profound knowledge of

* fabrizio.cutolo@endocas.org state-of-the-art in AR technology in order to find the most suitable solution for each clinical application to be conducted.

In this regard there are two aspects worthy of being studied. First one is related to the degree of alignment between virtual information and real scene. Unlike other fields of applications as consumer applications (on smartphones, tablets, some commercial AR glasses) in medical applications the accuracy in the alignment is crucial and depends on the tracking approach adopted and on the subsequent registration modality employed.

Furthermore the choice of the preferable tracking and registration methodology is related to the specific clinical application, thus another goal of the $\mathrm{PhD}$ work will consist in creating a sufficiently wide range of reliable technical solutions sharing some hardware and software common characteristics but differentiating on others. All proposed solutions will ideally have to be inexpensive and user-friendly to be successfully introduced in the clinical practice. The second key aspect that has a huge impact on the actual efficacy of AR applications is related to the ergonomics of the device. More in detail the ergonomics in rendering the virtual information influences AR usability in a clinical environment and strongly affects the physicians' attitude towards use and the actual acceptance of AR technology in medicine.

\section{Research Material and Methods}

As previously mentioned the most interesting applications comprising $\mathrm{AR}$ in medicine are related to the potential improvements in surgical navigation. By means of a variety of possible display modalities AR systems can act as surgical guidance, providing the ability to view hidden anatomies or completely virtual structures perfectly aligned with the patient [2].
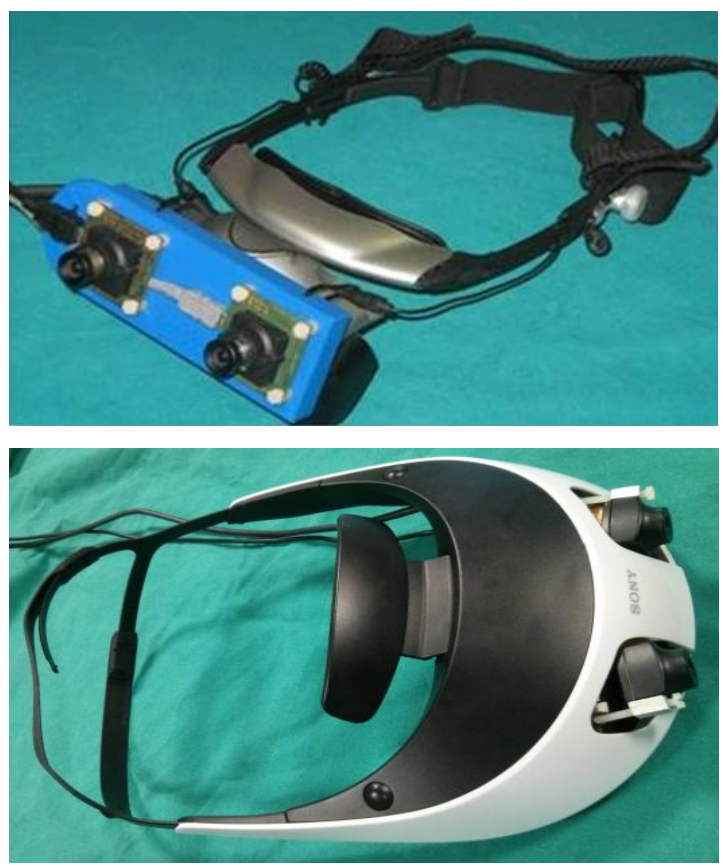

Figure 1: First model of the video see-through HMD (top image) and latest model (bottom image). 
Depending on the specific application, the augmented scene can be presented on displays of different kind of electronic devices (traditional PC, laptop, tablet PC, mobile phone) [5],[6],[7],[8],[13],[14],[15],[18],[19], it can be generated by an external projector directly over the surgical space [4],[9],[11],[20], rendered by an integral videography display [21] or it can be provided directly in front of the eyes of the surgeon through a head-mounted display (video see-through or optic seethrough) [3],[10],[22].

The correct fusion between virtual data and real scene has to be achieved by a robust and reliable tracking modality that provides in real time the point of view and real object pose in the world reference system.

As regards the different tracking modalities, most of the aforementioned studies are marker-based and rely either on cumbersome external infrared localizers [5],[6],[7],[8], or on standard digital cameras and video-based methods [10],[11]. Other tracking modalities are based on more complex surfacebased tracking algorithms [4],[16].

\subsection{Display modality}

Wearable AR systems based on Head Mounted Displays (HMDs) [10], [22], provide the user with a natural point of view. This issue is of key significance for those medical tasks manually performed under surgeon's direct vision (palpation, introduction of biopsy needle, open surgery, etc.).

Taking into account this consideration I therefore opted for a stereoscopic video see-through HMD whose ergonomics well suited to the needs of my first clinical studies. In Figure 1 my first and latest HMD models used in the clinical applications are shown. The video see-through paradigm is illustrated in Figure 2: real-world views are grabbed by two external cameras fixed in front of the visor and in correspondence of the user's eyes so to emulate hisher real sight. The captured images, after compensation of the radial distortion, are screened as backgrounds of the virtual scene on the corresponding display; finally the virtual objects are coherently added to the scene and observed by a couple of virtual cameras whose projective parameters and whose poses must mimic the real ones (see Tracking and Registration Modality section). In this case, unlike optic seethrough systems, it is not necessary to know the exact relative pose between displays and eyes to ensure a sufficiently accurate augmentation, fundamental precondition for its correct use in a clinical application [23].

\subsection{Tracking and Registration Modality}

The accurate and robust merging between real-world views and computer-generated objects is achieved if virtual scene is rendered with:

Virtual camera projection model $\sim=$ Real one (Camera calibration) Virtual cameras relative pose $\sim=$ Real one (Camera calibration) Virtual object poses $\sim=$ Real ones (Registration)

Camera calibration features the identification of both cameras' intrinsic parameters, their radial distortion model and the relative pose between them; this process is carried out before running the AR application. The registration process instead allows the estimation of the geometric relation (position and orientation) between reference objects and the cameras.

So far I have opted for a localizer-free marker-based approach leaving the alternative tracking methods, mainly feature-based tracking and hybrid tracking methods to be considered at a later stage of the $\mathrm{PhD}$ work.

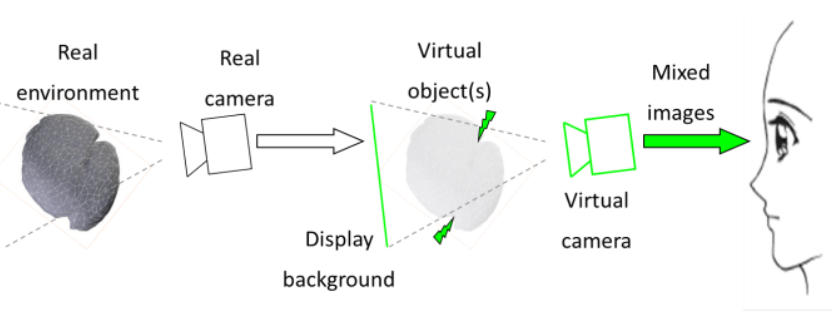

Figure 2: Video see-through paradigm.

\section{Results to date AND Discussion}

My initial solution features a localizer-free registration and takes into account key considerations regarding its actual applicability in a clinical scenario. The coherent fusion between virtual information and real-world views, used as backgrounds, is achieved through a real-time marker-based rigid registration method. Thanks to the results obtained in terms of system accuracy and robustness I managed to carry out a series of clinical in-vitro studies (maxillofacial surgery, orthopedics surgery), obtaining promising results in terms of system precision, usability and ergonomics.

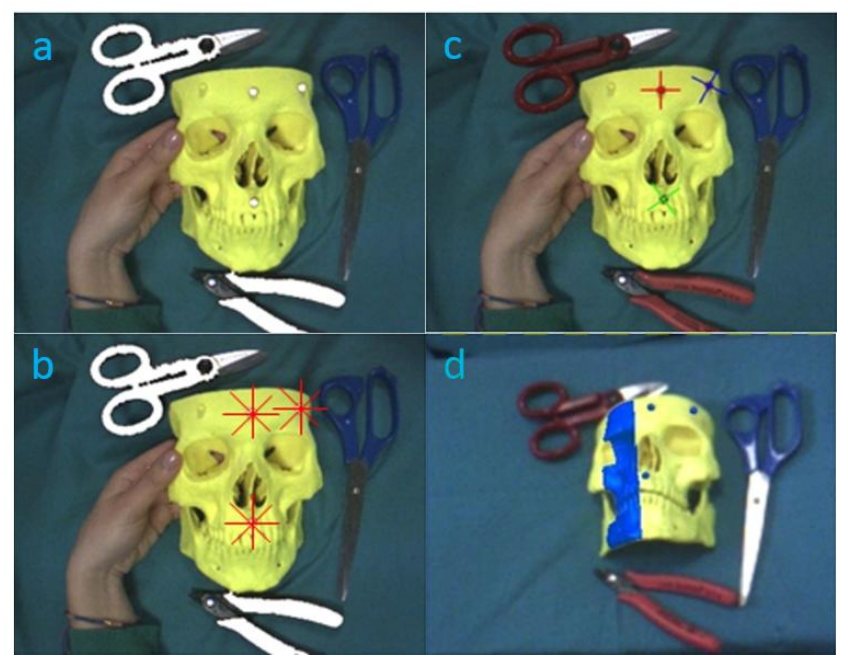

Figure 3: Machine vision methods applied on a single camera frame: a) Color Segmentation; b) Shape Recognition; c) Stereo Matching; d) Marker-based registration with pose refinement.

\subsection{Technical Advancements to Date}

A technical paper entitled "Robust and Accurate Algorithm for Stereoscopic Augmented Reality with Three Indistinguishable Markers" was recently submitted to the IEEE TVCG [24]. In the submitted work a marker-based video registration method which relies, as markers, on three indistinguishable monochromatic spheres is proposed. More in detail, use of 3 markers (minimum set of fiducial markers for our goal) is intended to limit the logistic payload for setup in the surgical room. Our system does not need for an external infrared tracker or electromagnetic field generator for real time registration since the HMD serves both as a frame-grabber and a localizer.

Stereo matching problem and markers tracking is achieved with the application of multiple machine vision methods as depicted in Figure 3 . 

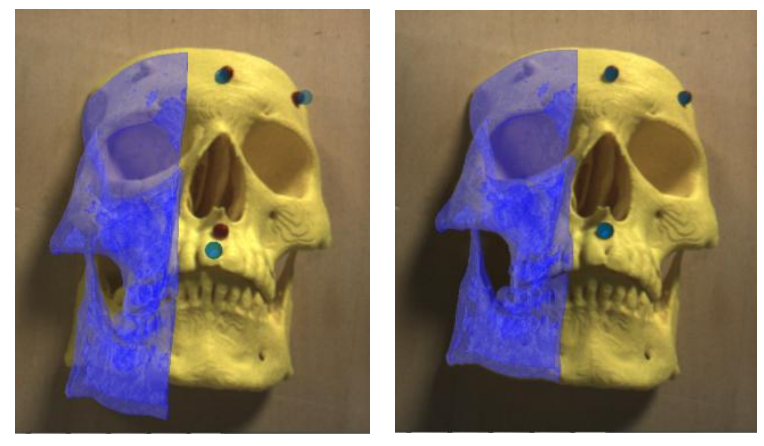

Figure 4: Results of the video-based registration applied on the left camera view using pre-determined calibration data before (Left image) and after (Right image) camera pose refinement.

Furthermore, with regard to the alignment problem, the proposed solution can provide a sub-pixel coherence between real-world views and virtual data thanks to the minimization of a cost function related to the back-projection error that refines separately each camera's pose (Figure 4).

The strategy of optimizing each camera's pose does not need a perfectly calibrated stereoscopic system and has been successfully tested on a new HMD with cameras with unfixed vergence (bottom image in Figure 1); the proposed system, which includes a lightweight vergence adjuster, yields high video registration accuracy without the need of frequent calibrations but just using pre-determined calibration data. The solution was described on a work entitled "HMD Video See Though AR with Unfixed Cameras Vergence" which was recently accepted as poster paper at ISMAR 2014 symposium [25].

\subsection{Maxillofacial surgery clinical study}

In the first clinical study we tested the video see-through HMD as aid in the execution of maxillofacial surgery on a physical replica of a human skull. The in vitro study was performed in 2013 in collaboration with a group of surgeons from the Maxillofacial Surgery Unit of the S. Orsola-Malpighi University Hospital of Bologna (Italy); primary goal of the work was to perform a feasibility and validation study of our localiser-free AR system in assisting waferless maxillary repositioning (Figure 5).

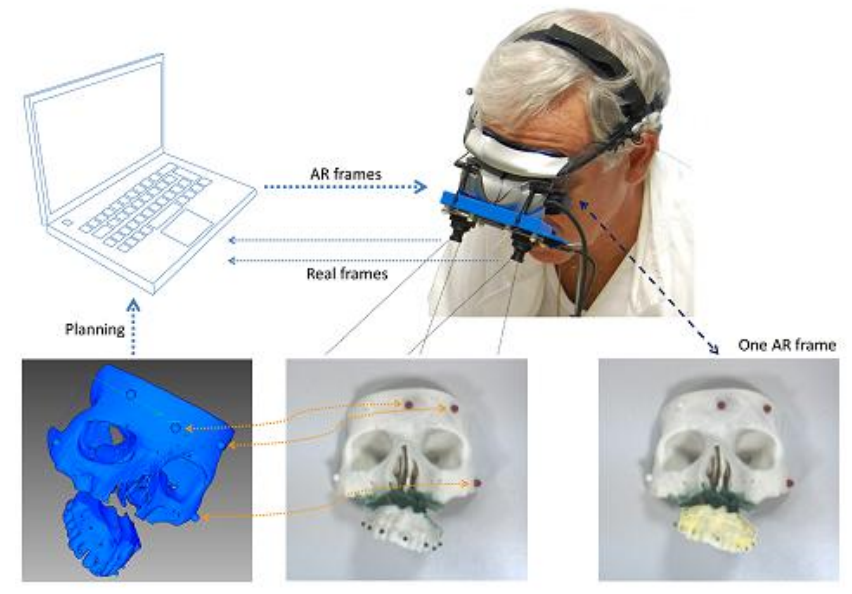

Figure 5: In vitro feasibility study of AR in assisting bone repositioning after LeFort 1 osteotomy.
Results in terms of geometric distances between obtained positions of some validation points over the maxilla (measured using a standard navigation system) and their expected positions (defined during planning) were very promising $(1.70 \pm 0.51 \mathrm{~mm})$. Further, results in terms of actual usability and ergonomics suggested that the proposed solution can potentially be extended to aid in many maxillofacial orthognathic procedures. The work herein introduced was illustrated within an article just accepted by the Journal of Cranio-Maxillofacial Surgery with the title "Augmented reality as an aid in maxillofacial surgery: Validation of a wearable system allowing maxillary repositioning" [26].

\subsection{Orthopedic surgery clinical study}

Another in vitro study is currently being completed together with surgeons of the 1st Orthopedic Division of the University of Pisa. In particular we are now testing the wearable AR system as aid in the task of reaching an intracorporeal target through percutaneous approach (i.e. percutaneous transpedicular vertebroplasty) avoiding the employment of ionizing radiation during the procedure.

Several tests were initially conducted to evaluate the ergonomics of the device and the most functional way of displaying the virtual guide to better assist the surgeon during needle insertion (Figure 6).

Preliminary results in terms of insertion accuracy into the vertebral body during percutaneous vertebroplasty on a spine phantom have been lately presented at the CAOS 2014 meeting (Computer Assisted Orthopedic Surgery) as poster presentation.
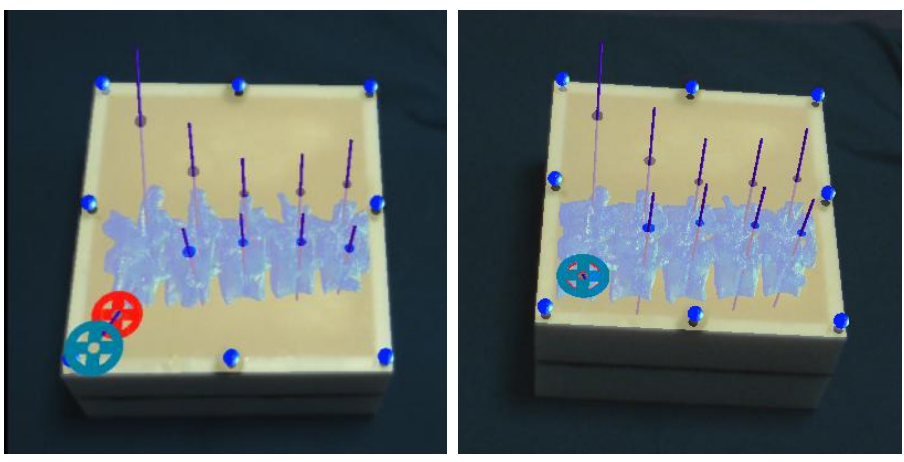

Figure 6: In vitro feasibility study of $\mathrm{AR}$ in assisting percutaneous transpedicular vertebroplasty.

\subsection{AR magnetic guidance of endovascular device}

Recently a third study has been accepted as contributed paper at the 36th Annual International IEEE EMBC Conference [27]; in this preliminary study I investigated, in an in vitro setup, the employment of the wearable AR system in providing the ideal path to be followed in guiding magnetic endovascular devices through an external magnet (a challenging and interesting new approach for endovascular device propulsion).

\section{EXPECTED CONTRIBUTION AND FUTURE WORKS}

Future works will comprise both technical improvements and additional clinical studies. On the technical side I am currently working on further increasing the accuracy and robustness of the tracking methods. In this regard I am envisioning advancements in the marker detection by working on the feature extraction procedure, by modifying the chromatic properties of the markers and $\backslash$ or the environment lighting: adaptive color segmentation using dynamic thresholds; use of fluorescent dyes; use of polarizing filters. 
In addition I might apply the methods for the accurate estimation of camera poses to other stereoscopic devices like binocular endoscopes or binocular microscopes to be tested on other surgical procedures.

With respect to the next clinical studies together with the surgeons I am preparing the protocol for the maxillofacial clinical trial and concluding the orthopedic surgery in vitro study.

\section{REFERENCES}

[1] P. Milgram and F. Kishino, "A Taxonomy of Mixed Reality Visual-Displays," Ieice Transactions on Information and Systems, vol. E77d, pp. 1321-1329, Dec 1994.

[2] M. Kersten-Oertel, et al., "The state of the art of visualization in mixed reality image guided surgery," Computerized Medical Imaging and Graphics, vol. 37, pp. 98-112, Mar 2013.

[3] V. Ferrari, et al., "A 3-D mixed-reality system for stereoscopic visualization of medical dataset," IEEE Trans Biomed Eng, vol. 56, pp. 2627-33, Nov 2009.

[4] R. Marmulla, et al., "An augmented reality system for imageguided surgery," Int J Oral Maxillofac Surg, vol. 34, pp. 594-6, Sep 2005.

[5] R. A. Mischkowski, et al., "Application of an augmented reality tool for maxillary positioning in orthognathic surgery - a feasibility study," J Craniomaxillofac Surg, vol. 34, pp. 478-83, Dec 2006.

[6] M. J. Zinser, et al., "Computer-assisted orthognathic surgery: waferless maxillary positioning, versatility, and accuracy of an imageguided visualisation display," Br J Oral Maxillofac Surg, vol. 51, pp. 827 33, Dec 2013.

[7] M. Caversaccio, et al., "Augmented reality endoscopic system (ARES): preliminary results," Rhinology, vol. 46, pp. 156-8, Jun 2008.

[8] D. Inoue, et al., "Preliminary study on the clinical application of augmented reality neuronavigation," J Neurol Surg A Cent Eur Neurosurg, vol. 74, pp. 71-6, Mar 2013.

[9] M. Mahvash and L. Besharati Tabrizi, "A novel augmented reality system of image projection for image-guided neurosurgery," Acta Neurochir (Wien), vol. 155, pp. 943-7, May 2013.

[10] Y. Abe, et al., "A novel 3D guidance system using augmented reality for percutaneous vertebroplasty," Journal of Neurosurgery-Spine, vol. 19, pp. 492-501, Oct 2013.

[11] J. R. Wu, et al., "Real-time advanced spinal surgery via visible patient model and augmented reality system," Comput Methods Programs Biomed, vol. 113, pp. 869-81, Mar 2014.

[12] J. Fritz, et al., "MR-Guided Vertebroplasty With Augmented Reality Image Overlay Navigation," Cardiovasc Intervent Radiol, Apr 11 2014.

[13] J. Kowalczuk, et al., "Real-time three-dimensional soft tissue reconstruction for laparoscopic surgery," Surg Endosc, vol. 26, pp. 34137, Dec 2012.

[14] E. Marzano, et al., "Augmented reality-guided artery-first pancreatico-duodenectomy," J Gastrointest Surg, vol. 17, pp. 1980-3, Nov 2013.

[15] S. Ieiri, et al., "Augmented reality navigation system for laparoscopic splenectomy in children based on preoperative CT image using optical tracking device," Pediatric Surgery International, vol. 28, pp. 341-346, Apr 2012.
[16] N. Haouchine, et al., "Deformation-based augmented reality for hepatic surgery," Stud Health Technol Inform, vol. 184, pp. 182-8, 2013.

[17] B. J. Dixon, et al., "Surgeons blinded by enhanced navigation: the effect of augmented reality on attention," Surg Endosc, vol. 27, pp. 454-61, Feb 2013.

[18] M. Muller, et al., "Mobile augmented reality for computerassisted percutaneous nephrolithotomy," International Journal of Computer Assisted Radiology and Surgery, vol. 8, pp. 663-675, Jul 2013.

[19] P. Mezzana, et al., "Augmented Reality in Oculoplastic Surgery: First iPhone Application," Plastic and Reconstructive Surgery, vol. 127, pp. 57e-58e, Mar 2011.

[20] K. Gavaghan, et al., "Evaluation of a portable image overlay projector for the visualisation of surgical navigation data: phantom studies," International Journal of Computer Assisted Radiology and Surgery, vol. 7, pp. 547-556, Jul 2012.

[21] H. E. Liao, et al., "3-D Augmented Reality for MRI-Guided Surgery Using Integral Videography Autostereoscopic Image Overlay," Ieee Transactions on Biomedical Engineering, vol. 57, pp. 1476-1486, Jun 2010.

[22] L. Johnson, et al., "Depth perception of stereo overlays in image-guided surgery," Medical Imaging 2004: Image Perception, Observer Performance, and Technology Assessment, vol. 5372, pp. $263-$ $272,2004$.

[23] F. Kellner, et al., "Geometric Calibration of Head-Mounted Displays and its Effects on Distance Estimation," Ieee Transactions on Visualization and Computer Graphics, vol. 18, pp. 589-596, Apr 2012.

[24] F.Cutolo, C.Freschi, S.Mascioli, P.Parchi, M.Ferrari and V.Ferrari, "Robust and Accurate Algorithm For Stereoscopic Augmented Reality With Three Indistinguishable Markers," Submitted to IEEE Transaction on Visualization and Computer Graphics, 2014.

[25] V. Ferrari, F.Cutolo, E.M. Calabrò, M.Ferrari, "HMD Video See Though AR with Unfixed Cameras Vergence," ISMAR 2014 symposium, 2014.

[26] G. Badiali, V.Ferrari, F. Cutolo, C. Freschi, D. Caramella, A. Bianchi and C. Marchetti, "Augmented reality for maxillofacial surgery: validation of a wearable system for maxillary repositioning," Submitted to Journal of Cranio-Maxillofacial surgery, 2014.

[27] S. Parrini, F. Cutolo, C. Freschi, M. Ferrari, V. Ferrari, "Augmented Reality System for Freehand Guide of Magnetic Endovascular Devices," in Accepted Proceedings at 36th Annual International IEEE EMBS Chicago, 2014. 